\title{
Split Octonions and Maxwell Equations
}

\section{P. NuROWSKI*}

Instytut Fizyki Teoretycznej, Uniwersytet Warszawski, Hoża 69, 00-681 Warszawa, Poland

(Received June 15, 2009; in final form October 5, 2009)

A formulation of the Maxwell equations in terms of the split octonions is presented.

PACS numbers: 03.50.-z, 03.50.De

\section{Introduction}

A well known multiplication rule for the imaginary octonions [1], depicted schematically on the Fano plane,

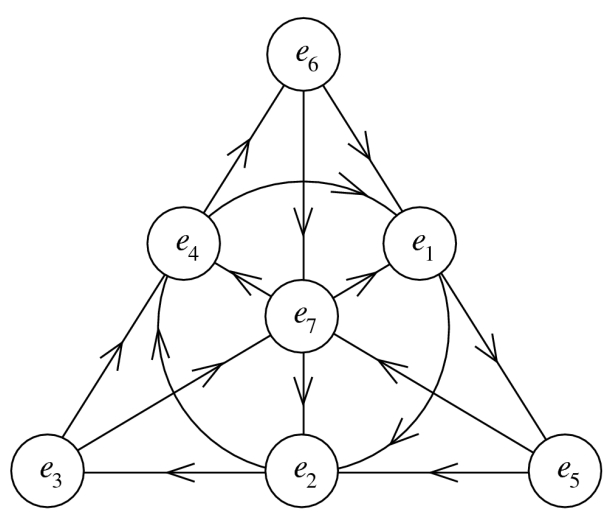

may be generalized to the following multiplication table:

TABLE

\begin{tabular}{c|c|c|c|c|c|c|c}
\hline \hline & $e_{1}$ & $e_{2}$ & $e_{3}$ & $e_{4}$ & $e_{5}$ & $e_{6}$ & $e_{7}$ \\
\hline$e_{1}$ & -1 & $e_{4}$ & $e_{7}$ & $-e_{2}$ & $e_{6}$ & $-e_{5}$ & $-e_{3}$ \\
$e_{2}$ & $-e_{4}$ & -1 & $e_{5}$ & $e_{1}$ & $-e_{3}$ & $e_{7}$ & $-e_{6}$ \\
$e_{3}$ & $-e_{7}$ & $-e_{5}$ & $\mp 1$ & $e_{6}$ & $\pm e_{2}$ & $\mp e_{4}$ & $\pm e_{1}$ \\
$e_{4}$ & $e_{2}$ & $-e_{1}$ & $-e_{6}$ & -1 & $e_{7}$ & $e_{3}$ & $-e_{5}$ \\
$e_{5}$ & $-e_{6}$ & $e_{3}$ & $\mp e_{2}$ & $-e_{7}$ & $\mp 1$ & $\pm e_{1}$ & $\pm e_{4}$ \\
$e_{6}$ & $e_{5}$ & $-e_{7}$ & $\pm e_{4}$ & $-e_{3}$ & $\mp e_{1}$ & $\mp 1$ & $\pm e_{2}$ \\
$e_{7}$ & $e_{3}$ & $e_{6}$ & $\mp e_{1}$ & $e_{5}$ & $\mp e_{4}$ & $\mp e_{2}$ & $\mp 1$
\end{tabular}

In this table the upper sign corresponds to the multiplication of the imaginary octonions and the lower sign to the multiplication of the imaginary split octonions. Via the above multiplication rules the 8-dimensional real vector space

$\operatorname{Span}_{\mathbb{R}}\left\{1, e_{1}, e_{2}, e_{3}, e_{4}, e_{5}, e_{6}, e_{7}\right\}$

* e-mail: nurowski@fuw.edu.pl gets equipped with the structure of two (nonassociative) algebras with unit 1 . These two algebras are called octonions for the upper sign in the table and the split octonions for the lower sign.

In this letter we will use a vector-valued (split) octonion $\boldsymbol{Q}=\left(e_{1}, e_{2}, e_{4}\right)$ and its corresponding vector-valued (split)octonion $e_{7} \boldsymbol{Q}=\left(e_{7} e_{1}, e_{7} e_{2}, e_{7} e_{4}\right)=\left(e_{3}, e_{6}, e_{5}\right)$.

\section{Model}

Consider now two vector fields on $\mathbb{R}^{4}$ :

$$
\boldsymbol{E}: \mathbb{R}^{4} \rightarrow \mathbb{R}^{3}, \quad(t, x, y, z) \mapsto \boldsymbol{E}=\left(E_{x}, E_{y}, E_{z}\right)
$$

and

$$
\boldsymbol{B}: \mathbb{R}^{4} \rightarrow \mathbb{R}^{3}, \quad(t, x, y, z) \mapsto \boldsymbol{B}=\left(B_{x}, B_{y}, B_{z}\right) .
$$

Define

$$
\begin{aligned}
F & =E_{x} e_{1}+E_{y} e_{2}+E_{z} e_{4}+B_{x} e_{3}+B_{y} e_{6}+B_{z} e_{5} \\
& =\boldsymbol{E} \boldsymbol{Q}+\boldsymbol{B}\left(e_{7} \boldsymbol{Q}\right) \\
& =\left(E_{x}+e_{7} B_{x}\right) e_{1}+\left(E_{y}+e_{7} B_{y}\right) e_{2}+\left(E_{z}+e_{7} B_{z}\right) e_{4} \\
& =\left(\boldsymbol{E}+e_{7} \boldsymbol{B}\right) \boldsymbol{Q} .
\end{aligned}
$$

and

$$
\partial=e_{1} \partial_{x}+e_{2} \partial_{y}+e_{4} \partial_{z}+e_{7} \partial_{t} .
$$

Then using the above multiplication table and the standard notation of vector calculus in $\mathbb{R}^{3}$ we have

$$
\begin{aligned}
\partial F & =-\boldsymbol{\nabla} \boldsymbol{E}+\left(\boldsymbol{\nabla} \times \boldsymbol{E} \mp \frac{\partial \boldsymbol{B}}{\partial t}\right) \boldsymbol{Q} \\
& +\left(-\boldsymbol{\nabla} \times \boldsymbol{B}+\frac{\partial \boldsymbol{E}}{\partial t}\right)\left(e_{7} \boldsymbol{Q}\right)+(\boldsymbol{\nabla} \boldsymbol{B}) e_{7} .
\end{aligned}
$$

Thus we see that if we choose the split octonions (lower $\operatorname{sign})$, then

$$
\partial F=0
$$

is equivalent to the equations

$$
\begin{aligned}
& \boldsymbol{\nabla} \boldsymbol{E}=0, \quad \boldsymbol{\nabla} \times \boldsymbol{E}=-\frac{\partial \boldsymbol{B}}{\partial t}, \\
& \boldsymbol{\nabla} \boldsymbol{B}=0, \quad \boldsymbol{\nabla} \times \boldsymbol{B}=\frac{\partial \boldsymbol{E}}{\partial t} .
\end{aligned}
$$




\section{Remarks}

Several remarks are in order:

Remark 0.1. Why the Nature prefers the split octonions rather than the octonions for the electromagnetism?

Remark 0.2. It is interesting to note that $F$ defined above is not a generic imaginary split octonion. If we were to choose a generic imaginary split octonion

$$
F=E_{x} e_{1}+E_{y} e_{2}+E_{z} e_{4}+B_{x} e_{3}+B_{y} e_{6}+B_{z} e_{5}+S e_{7} \text {, }
$$

where $S=S(t, x, y, z)$ was arbitrary function on $\mathbb{R}^{4}$, then

$$
\begin{aligned}
\partial F & =\left(-\boldsymbol{\nabla} \boldsymbol{E}+\frac{\partial S}{\partial t}\right)+\left(\boldsymbol{\nabla} \times \boldsymbol{E}+\frac{\partial \boldsymbol{B}}{\partial t}\right) \boldsymbol{Q} \\
& +\left(-\boldsymbol{\nabla} \times \boldsymbol{B}+\frac{\partial \boldsymbol{E}}{\partial t}-\boldsymbol{\nabla} S\right)\left(e_{7} \boldsymbol{Q}\right)+(\boldsymbol{\nabla} \boldsymbol{B}) e_{7} .
\end{aligned}
$$

In such case $\partial F=0$ would correspond to

$$
\begin{aligned}
& \boldsymbol{\nabla} \boldsymbol{E}=\frac{\partial S}{\partial t}, \quad \boldsymbol{\nabla} \times \boldsymbol{E}+\frac{\partial \boldsymbol{B}}{\partial t}=0, \\
& \boldsymbol{\nabla} \boldsymbol{B}=0, \quad-\boldsymbol{\nabla} \times \boldsymbol{B}+\frac{\partial \boldsymbol{E}}{\partial t}=\boldsymbol{\nabla} S .
\end{aligned}
$$

These equations for $(\boldsymbol{E}, \boldsymbol{B})$ could be then interpreted as the Maxwell equations for electromagnetic field $(\boldsymbol{E}, \boldsymbol{B})$ generated by the charge density $\rho=\partial S / \partial t$ and the cur- rent density $j=\nabla S$. Let us note that to get the magnetic charge densities and magnetic currents we would need to introduce the generic (not purely imaginary) split octonion $F$.

Remark 0.3. Now the story is quite puzzling: an automorphism $\sigma$ of the split octonions is an element of the noncompact real form of the exceptional Lie group $G_{2}$. Since $\sigma(\partial F)=\sigma(\partial) \sigma(F)$, then, if $F$ satisfied the Maxwell equations $\partial F=0$, the transformed $\sigma(F)$ would satisfy the equations $\sigma(\partial) \sigma(F)=0$. But the transformed field $\sigma(F)$ is a general split octonion; the transformed derivative $\sigma(\partial)$ also is. The physical interpretation of the $\sigma$-induced transformation on the space time coordinates $(t, x, y, z)$ and the electromagnetic field $(\boldsymbol{E}, \boldsymbol{B})$ would be interesting.

Remark 0.4. After the acceptance of this note in this journal I received a message from Prof. Murat Gunaydin, who send me a copy of Refs. [2,3], in which a similar formulation of the Maxwell [3] (respectively Yang-Mills [2]) equations in terms of the split octonions was presented.

\section{References}

[1] J.C. Baez, The Octonions, Bull. Amer. Math. Soc. 39, 145 (2002).

[2] A.M. Buoncristiani, J. Math. Phys. 14, 849 (1973).

[3] M. Gunaydin, Ph.D. Thesis, Yale University, 1973. 